\title{
A Practical Method for the Determination of Time Step in Non-Orthogonal FDTD
}

\author{
R. Nilavalan, I.J. Craddock and C.J. Railton \\ Centre for Communications Research, University of Bristol, \\ Merchant Venturers Building, Woodland Road, Bristol, BS8 1UB.
}

\begin{abstract}
The Non-Orthogonal FDTD algorithm is well-suited to the modelling of curved structures, however users of the technique frequently have difficulty determining an appropriate time step for the algorithm. This contribution presents a new method which is shown to be both a practical and reliable technique for determining the time step.
\end{abstract}

\section{Introduction}

The original Finite-Difference Time-Domain (FDTD) method is a full-wave, timedomain electromagnetic analysis technique that is particularly attractive for broadband structures. A generalisation of the original method to Non-Orthogonal meshes [1, 2, 3] is better-suited to problems with curved boundaries.

Like the conventional FDTD method, this Non-Orthogonal algorithm requires the choice of a time step, $\Delta t$, at the start of each run. The maximum allowable time step depends on the mesh sizes and angles - its rigorous calculation requires the consideration of the eigenvalues of the algorithm update equations [2, 3].

A full eigenvalue analysis is however not a practical technique for finding a time step for each Non-Orthogonal FDTD problem, as the computation required is prohibitive. On the other hand, approximate methods [4], which attempt to find $\Delta t$ using a generalised Courant condition, do not (as this paper shows) yield a reliable estimate.

The absence of a practical and reliable method to calculate the algorithm time step is a major problem for potential users of the Non-Orthogonal FDTD method.

This contribution presents a novel method of determining a time step for a given NonOrthogonal FDTD mesh, without resorting to the computational expense of a full eigenvalue analysis. The reliability of this new method is demonstrated by considering some typical, complex mesh configurations.

\section{Existing Techniques for Time Step Estimation}

Non-Orthogonal FDTD may be described by a system of matrix-vector equations [2,3]:

An iteration step (where new values of contravariant fields are calculated):

$$
\dot{\mathbf{B}}=\mathbf{C}_{e} \mathbf{E} \quad \dot{\mathbf{D}}=\mathbf{C}_{h} \mathbf{H}
$$

An interpolation step (where covariant components are calculated using the metric tensor $g_{i j}$ ):

$$
\begin{aligned}
& E_{i}=\sum_{j} \frac{g_{i j}}{\varepsilon \sqrt{g}} D_{j} \Rightarrow \mathbf{E}=\mathbf{M}_{\varepsilon} \mathbf{D} \\
& \text { and, similarly, } \mathbf{H}=\mathbf{M}_{\mu} \mathbf{B}
\end{aligned}
$$

- where $\mathbf{E}$ and $\mathbf{H}$ are vectors consisting of all the covariant field values in the algorithm, $\mathbf{B}$ and $\mathbf{D}$ are the contravariant components and $\mathbf{C}_{e}$ and $\mathbf{C}_{h}$ are matrices that implement the curl operation. $\mathbf{M}_{\varepsilon}$ and $\mathbf{M}_{\mu}$ are matrices that describe material properties and the interpolation that yields the necessary covariant components. 
For the Non-Orthogonal algorithm to be stable, the time step, $\Delta t$ (used to discretise equation 1 in time) must be less than $\gamma$ [3], where:

$$
\gamma=\frac{2}{\sqrt{\lambda_{m}}}
$$

- and $\lambda_{m}$ is the largest eigenvalue of $\mathbf{M}=\mathbf{M}_{\varepsilon} \mathbf{C}_{h} \mathbf{M}_{\mu} \mathbf{C}_{e}$.

If, for example, the FDTD mesh consists of $100 \times 100 \times 100$ nodes (which is not unrealistic), the $\mathbf{C}$ and $\mathbf{M}$ matrices are square with dimensions of $10^{6} \times 10^{6}$ (although sparse). Although fast algorithms are available to compute the maximum eigenvalue of sparse matrices, the size of $\mathbf{M}$ makes equation (4) impractical for determining $\Delta t$.

If, however, the interpolation step (equations 2 and 3) is ignored then the generalised Courant criteria applies and can be used to find the maximum allowable time step. The generalised Courant criteria is given by [4]:

$$
\Delta t \leq \frac{1}{c \sqrt{\sum_{\substack{i=1 \\ j=1}}^{3} g^{i j}}}
$$

where $\left[\mathrm{g}^{i j}\right]=\left[g_{i j}\right]^{-1}$.

This rapid technique has employed by several authors to find the time step [4, 5]. However, as shown in section 4, in neglecting the interpolation in the Non-Orthogonal algorithm, it yields an unreliable time step that is likely to result in numerical instability.

The two methods available in the literature to date are therefore either impractical or unreliable. This contribution therefore presents a compromise method that is practical and yet, even in complex meshes, reliable.

\section{A New Technique for Time Step Estimation}

This section describes a technique that, for all the Non-Orthogonal FDTD problems considered by the authors to date, yields a safe value of time step without resorting to an impractical full eigenvalue analysis. The method proposed is:

i. Employ the generalised Courant condition (5) to the mesh. Find the location $\boldsymbol{i}$ which yields the minimum time step from this criterion.

ii. Form a sub-matrix $\mathbf{M}_{\boldsymbol{i}}$ with coefficients calculated from a few cells surrounding location $\boldsymbol{i}$ (e.g. $5 \times 5 \times 5=125$ cells as opposed to $100 \times 100 \times 100=10^{6}$ cells).

iii. Calculate the maximum eigenvalue $\lambda_{m i}$ of matrix $\mathbf{M}_{i}$.

iv. Find $\gamma_{i}$ from $\lambda_{m i}$, using eq. (4). Use a factor of 0.9 to estimate the time step from $\gamma_{i}$.

This technique is an approximation, since it assumes that step (i) yields the cells responsible for the time step limit and that step (iv) makes appropriate allowance for the fact that the largest eigenvalue of $\mathbf{M}_{\boldsymbol{i}}$ will not be identical to the largest eigenvalue of $\mathbf{M}$. Ultimately the validity of this approach must be determined by applying it to a varied selection of Non-Orthogonal meshes - as in the subsequent section.

\section{Demonstration of the New Technique for Time Step Estimation}

A variety of Non-Orthogonal meshes (Figures 1 to 4 ) were considered in order to validate the time step estimation approach. Table 1 summarises the results. 
- Mesh 1 was an orthogonal mesh generated for test purposes.

- Mesh 2, shown in Figure 1, is a mesh uniformly skewed to $\theta_{1}=55^{\circ}$ in the horizontal plane and orthogonal in all other planes.

- Mesh 3, which is too complex to draw satisfactorily, is non-uniformly skewed in all three planes, with varying angles $52^{\circ}<\theta_{1}<90^{\circ}, 39^{\circ}<\theta_{2}<90^{\circ}$ and $38^{\circ}<\theta_{3}<90^{\circ}$.

- Mesh 4 (Figure 2) and 5 are meshes for a two element conformal antenna array test structure (Mesh 5 is similar to Mesh 4 but more smoothly-curved).

- Mesh 6 (Figure 3) is a cylindrical resonator problem, using very small cell angles.

Table 1 shows that the time-step obtained from the Generalised Courant Condition is usually unreliable; it alternates between being very optimistic (e.g. Mesh 3) and being slightly pessimistic (e.g. Mesh 4).

In contrast, the new technique proposed herein works reliably. The value of time step yielded by the technique (with a matrix $\mathbf{M}_{i}$ formed using a $5 \times 5 \times 5$ cell volume around location $\boldsymbol{i}$ ) remains close to, yet beneath, the maximum value at all times.

\section{Conclusions}

It has been proposed that the time step for a Non-Orthogonal FDTD problem may be calculated from an eigenvalue analysis of just a few cells in the mesh.

Unlike an eigenvalue analysis of the full FDTD algorithm, this technique is eminently useable, while, unlike the Generalised Courant Condition, it is reliable - as demonstrated here using a varied sample of realistic test problems. 


\section{References}

[1] Holland, R.: 'Finite-Difference Solution of Maxwell's Equations in Generalised Non-Orthogonal Co-ordinates', IEEE Transactions on Nuclear Science, 1983, 30, pp 4586-4591.

[2] Schuhmann, R., and Weiland, T.: 'Stability of the FDTD algorithm on Nonorthogonal Grids Related to the Spatial Interpolation Scheme', IEEE Transactions on Magnetics, 1998, 34, pp 2751-2754.

[3] Gedney, S.D., and Roden, J.A.: 'Numerical Stability of Non-orthogonal FDTD methods', IEEE Transactions on Antennas and Propagation, 2000, 48, pp. 231239.

[4] Lee, J.F., Palandech, R., and Mittra, R.: 'Modelling Three Dimensional Discontinuities in Wave Guides using Non-orthogonal FDTD Algorithm', IEEE Transactions on Microwave Theory and Techniques, 1992, 40, pp. 346-352.

[5] Chen, Q. and Fusco, V.F.: 'Three dimensional cylindrical co-ordinate finitedifference time-domain analysis of curved slotline', Proc. Second International Conf. Computation in Electromagnetics, 1994, pp 323-326. 
Tables

\begin{tabular}{|c|c|c|c|}
\hline Mesh & $\begin{array}{c}\Delta t \text { from Gen. Courant } \\
\text { Condition (5) }\end{array}$ & $\begin{array}{c}\Delta t \text { with technique } \\
\text { proposed herein }\end{array}$ & $\begin{array}{c}\text { Actual stable } \Delta t \text { from } \\
\text { numerical experiments }\end{array}$ \\
\hline 1 & $1.032 \mathrm{ps}$ & $0.969 \mathrm{ps}$ & $1.032 \mathrm{ps}$ \\
\hline 2 & $1.028 \mathrm{ps}$ & $0.727 \mathrm{ps}$ & $0.750 \mathrm{ps}$ \\
\hline 3 & $0.999 \mathrm{ps}$ & $0.546 \mathrm{ps}$ & $0.550 \mathrm{ps}$ \\
\hline 4 & $0.160 \mathrm{ps}$ & $0.173 \mathrm{ps}$ & $0.180 \mathrm{ps}$ \\
\hline 5 & $0.228 \mathrm{ps}$ & $0.258 \mathrm{ps}$ & $0.270 \mathrm{ps}$ \\
\hline 6 & $2.222 \mathrm{ps}$ & $2.037 \mathrm{ps}$ & $2.230 \mathrm{ps}$ \\
\hline
\end{tabular}

Table 1: Time Step Estimations 


\section{List of Figures}

Figure 1: Mesh 2: Skewed mesh for a Microstrip.

Figure 2: Mesh 4: Distorted mesh for two element antenna array.

Figure 3: Mesh 6: Resonator problem 
Figures

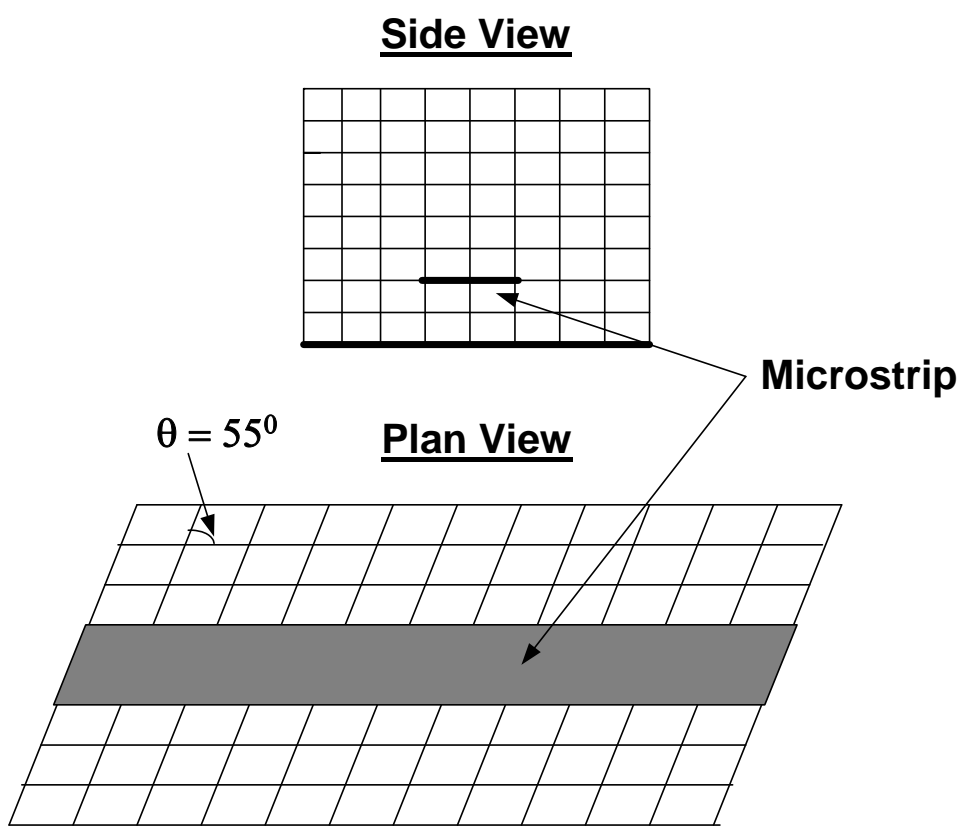

Figure 1: Mesh 2: Skewed mesh for a Microstrip.

orthogonal mesh

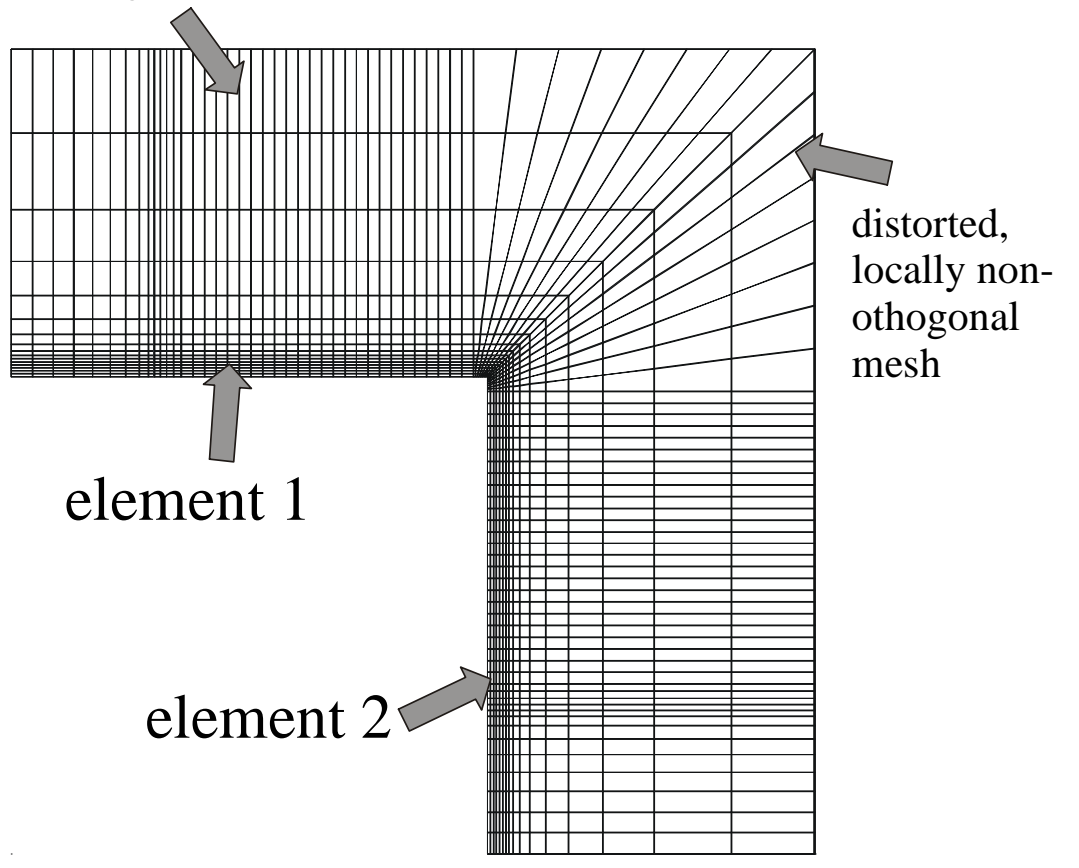

Figure 2: Mesh 4: Distorted mesh for two element antenna array. 
Absorbing boundaries

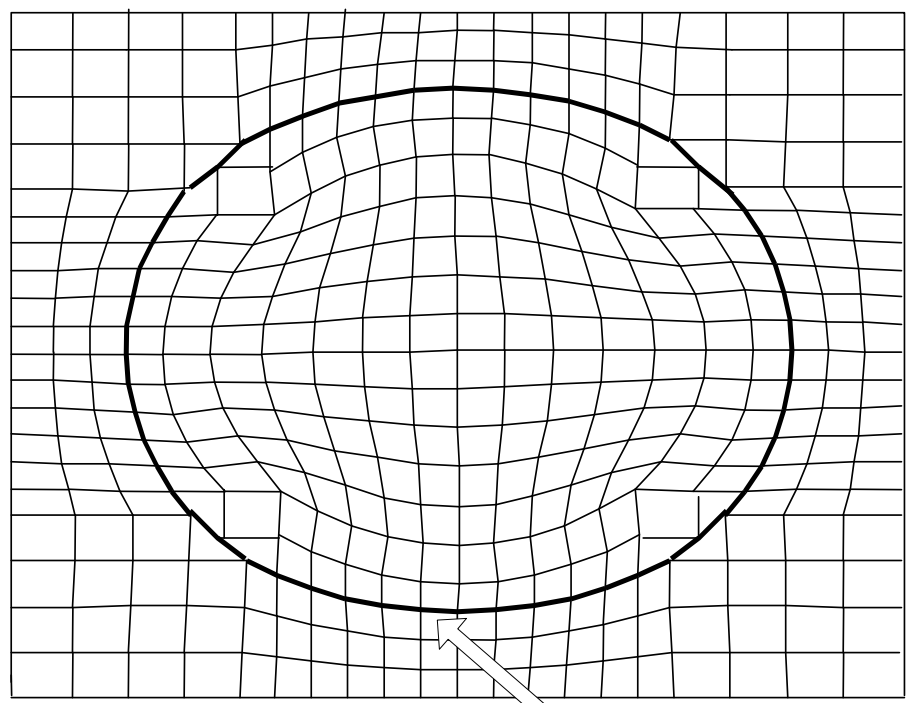

Resonator

Figure 3: Mesh 6: Resonator problem 